\title{
Fast quantum logic gates with trapped ions interacting with external laser and quantized cavity field beyond the Lamb-Dicke regime
}

\author{
S. Shelly Sharma ${ }^{1,2}$ and A. Vidiella-Barranco ${ }^{1}$ \\ (1) Instituto de Física "Gleb Wataghin", Universidade Estadual de \\ Campinas, 13083-970 Campinas SP Brazil \\ (2) Depto. de Física, Universidade Estadual de Londrina, 86040-370 \\ Londrina, PR Brazil \\ email : shelly@uel.br,vidiella@ifi.unicamp.br
}

\begin{abstract}
A scheme to implement quantum logic gates by manipulating trapped ions through interaction with monochromatic external laser field and quantized cavity field, beyond the Lamb-Dicke regime, is presented. Characteristic times, for implementing ionic state transitions using non-resont laser pulse or quantized cavity field, show a sharp decline for a relatively large Lamb-Dicke parameter value of $\eta_{L}=\eta_{c}=0.2$, and are seen to decrease further with increase in number of initial state vibrational quanta $m$.
\end{abstract}

Quantum computation is equivalent to performing a number of unitary operations on a multi qubit quantum system. A single qubit unitary gate and two-qubit controlled-NOT(CN) gate constitute a typical universal set for implementation of multi qubit logic gates [1]. Manipulation of trapped cold two-level ions interacting with laser field $[2,3]$, offers a mechanism for realizing unitary operations needed to construct quantum logic gates in Lamb-Dicke regime [4] and beyond [5,6]. Ions in a trap placed inside a high-Q cavity with a single mode quantized field, radiated by an external laser field [7], offer a much more versatile physical system. The advantage over ion-trap system comes from additional degrees of freedom and the constraints to which ionic motion is subjected to, due to quantization of the cavity field. The state transfer between cavity field mode and motional states of trapped ion has been suggested [8] and other experiments with ion trap placed inside a cavity have been proposed [9-11]. On the experimental side, a trapped ion has been used to probe the cavity field [12], and coherent coupling of an ion to the cavity field has been achieved [13]. For experimental realization of quantum logic gates involving several qubits, the ion state manipulation time is an extremely important factor. We propose the construction of a Hadamard gate, quantum phase gate, and controlled-NOT gate, through manipulation of internal and vibrational states of cold trapped ions placed inside a single mode high-Q cavity. Ionic state manipulation involves selective adjustment of laser-ion and quantized field-ion detuning parameters. Working in the region beyond the Lamb-Dicke regime $\left(\eta_{L}<1\right.$ and $\eta_{c}<1$, where $\eta_{L}$ and $\eta_{c}$ are Lamb-Dicke (LD) parameters relative to the external laser and cavity field respectively), we find considerable reduction in time needed to implement elementary qubit operations. Implementation of controlled-NOT gate in double Lamb-Dicke regime $\left(\eta_{L} \ll 1\right.$ and $\left.\eta_{c} \ll 1\right)$, has been proposed in ref. [14].

Consider a two-level ion confined in a trap placed inside a high-finesse cavity. The ion is radiated by the single mode cavity field of frequency $\omega_{c}$ and an external laser field of frequency $\omega_{L}$ and phase $\phi$. Entanglement of internal states of the ion, vibrational states of ionic center of mass, and the states of quantized cavity field, through interaction of ion with external laser and the cavity field, provides basic mechanism for qubit state rotations in Hilbert space. The system Hamiltonian, for the case when center of the trap is close to the node of cavity field standing wave, is given by [14]

$$
\begin{gathered}
\hat{H}=\hat{H}_{0}+\hat{H}_{i n t}, \\
\hat{H}_{0}=\hbar \nu\left(\hat{a}^{\dagger} \hat{a}+\frac{1}{2}\right)+\hbar \omega_{c} \hat{b}^{\dagger} \hat{b}+\frac{\hbar \omega_{0}}{2} \sigma_{z}, \\
\hat{H}_{i n t}=\hbar \Omega\left[\sigma_{+} \exp \left[i \eta_{L}\left(\hat{a}^{\dagger}+\hat{a}\right)-i\left(\omega_{L} t+\phi\right)\right]+h . c .\right] \\
+\hbar g\left(\sigma_{+}+\sigma_{-}\right)\left(\hat{b}^{\dagger}+\hat{b}\right) \sin \left[\eta_{c}\left(\hat{a}^{\dagger}+\hat{a}\right)\right],
\end{gathered}
$$

where $\hat{a}^{\dagger}(\hat{a})$ and $\hat{b}^{\dagger}(\hat{b})$ are creation(destruction) operators for vibrational phonon and cavity field photon respectively and $\omega_{0}$ the transition frequency of the two-level ion. The ion-phonon and ion-cavity coupling constants are $\Omega$ and $g$, whereas $\sigma_{k}(k=z,+,-)$ are the Pauli operators qualifying the internal state of the ion.

In the interaction picture, determined by unitary transformation $U_{0}(t)=\exp \left[-i \hat{H}_{0} t / \hbar\right]$, the Hamiltonian $\hat{H}_{I}$ reads as 


$$
\begin{aligned}
\hat{H}_{I}=\hbar \Omega\left[\sigma_{+} \hat{O}_{0}^{L} \exp \left[i\left(\delta_{0 L} t-\phi\right)\right]+h . c .\right] \\
+\hbar \Omega\left[\sigma_{+} \sum_{k=1}^{\infty}\left(i \eta_{L}\right)^{k} \hat{O}_{k}^{L} \hat{a}^{k} \exp \left[i\left(\left(\delta_{0 L}-k \nu\right) t-\phi\right)\right]+h . c .\right] \\
+\hbar \Omega\left[\sigma_{+} \sum_{k=1}^{\infty}\left(i \eta_{L}\right)^{k} \hat{a}^{\dagger k} \hat{O}_{k}^{L} \exp \left[i\left(\left(\delta_{0 L}+k \nu\right) t-\phi\right)\right]+h . c .\right] \\
+\hbar g\left[\sigma_{+} \hat{b}^{\dagger} \sum_{k=1,3, . .}^{\infty}\left(i^{k-1} \eta_{c}{ }^{k}\right) \hat{a}^{\dagger k} \hat{O}_{k}^{c} \exp \left[i\left(\delta_{0 c}+k \nu+2 \omega_{c}\right) t\right]+h . c .\right] \\
+\hbar g\left[\sigma_{+} \hat{b}^{\dagger} \sum_{k=1,3, . .}^{\infty}\left(i^{k-1} \eta_{c}{ }^{k}\right) \hat{O}_{k}^{c} \hat{a}^{k} \exp \left[i\left(\delta_{0 c}-k \nu+2 \omega_{c}\right) t\right]+h . c .\right. \\
+\hbar g\left[\sigma_{+} \hat{b} \sum_{k=1,3, . .}^{\infty}\left(i^{k-1} \eta_{c}{ }^{k}\right) \hat{O}_{k}^{c} \hat{a}^{k} \exp \left[i\left(\delta_{0 c}-k \nu\right) t\right]+h . c .\right] \\
+\hbar g\left[\sigma_{+} \hat{b} \sum_{k=1,3, . .}^{\infty}\left(i^{k-1} \eta_{c}{ }^{k}\right) \hat{a}^{\dagger k} \hat{O}_{k}^{c} \exp \left[i\left(\delta_{0 c}+k \nu\right) t\right]+h . c .\right]
\end{aligned}
$$

where $\delta_{0 L}=\omega_{0}-\omega_{L}, \delta_{0 c}=\omega_{0}-\omega_{c}$, and

$$
\hat{O}_{k}=\exp \left(-\frac{\eta^{2}}{2}\right) \sum_{p=0}^{\infty} \frac{(i \eta)^{2 p} \hat{a}^{\dagger p} \hat{a}^{p}}{p !(p+k) !} .
$$

The matrix element of diagonal operator $\hat{O}_{k}$ for a given vibrational state $m$ is given by

$$
\left\langle m\left|\hat{O}_{k}\right| m\right\rangle=\exp \left(-\frac{\eta^{2}}{2}\right) \sum_{p=0}^{m} \frac{\left(i \eta_{L}\right)^{2 p} m !}{p !(p+k) !(m-p) !} .
$$

We may note that the case where center of the trap lies close to the anti node of cavity standing wave field can be treated in an analogous fashion.

We next examine the construction of quantum logic gates namely Hadamard gate, phase gate, and ControlledNOT gate by proper choice of detuning parameters $\delta_{0 L}$ and $\delta_{0 c}$, and phase $\phi$ for ionic state manipulation. For this purpose, we consider the time evolution of the system for the special choices (i) $\delta_{0 L}=0$ that is the resonant laser field, (ii) $\delta_{0 L}=k \nu$, non resonant laser pulse, and (iii) $\delta_{0 c}=-k \nu$ where the quantized cavity field is resonant with $k^{t h}$ blue-shifted vibrational side band.

Case I. Resonant laser Pulse, $\delta_{0 L}=0$

In rotating wave approximation, the relevant part of Hamiltonian is

$$
\hat{H}_{1}=\hbar \Omega\left[\sigma_{+} \hat{O}_{0}^{L} \exp \left(-i \phi_{1}\right)+\sigma_{-} \hat{O}_{0}^{L} \exp \left(i \phi_{1}\right)\right] .
$$

We work in the basis $|g, m, n\rangle,|e, m, n\rangle$, where $m, n=0,1, . ., \infty$, denote the state of ionic vibrational motion and quantized cavity field, respectively. The state of the system at a time $t$, starting from initial states $|g, m, n\rangle$, and $|e, m, n\rangle$, is given respectively by

$$
|g, m, n\rangle \Rightarrow \cos \left(\Omega F_{m, m}^{L} t\right)|g, m, n\rangle-i \exp \left(-i \phi_{1}\right) \sin \left(\Omega F_{m, m}^{L} t\right)|e, m, n\rangle,
$$

and

$$
|e, m, n\rangle \Longrightarrow \cos \left(\Omega F_{m, m}^{L} t\right)|e, m, n\rangle-i \exp \left(i \phi_{1}\right) \sin \left(\Omega F_{m, m}^{L} t\right)|g, m, n\rangle,
$$

where the real matrix element $F_{m, m}^{L}=\left\langle m\left|\hat{O}_{0}^{L}\right| m\right\rangle$. In the approximation, $\eta_{L} \ll 1, F_{m, m}^{L}$ approaches 1 for all values of $m$, whereas for $\eta_{L}<1$ in the region beyond the Lamb-Dicke regime, the matrix element $F_{m, m}^{L}<1$.

Case II. Non resonant laser pulse, $\delta_{0 L}=k \nu, k=1,2, . . \infty$ 
The time evolution, in rotating wave approximation, is governed by the term

$$
\hat{H}_{2}=\hbar \Omega\left[\sigma_{+}\left(i \eta_{L}\right)^{k} \hat{O}_{k}^{L} \hat{a}^{k} \exp \left(-i \phi_{2}\right)+\sigma_{-}\left(-i \eta_{L}\right)^{k} \hat{a}^{\dagger k} \hat{O}_{k}^{L} \exp \left(i \phi_{2}\right)\right] .
$$

The initial states $|g, m, n\rangle$, and $|e, m, n\rangle$, evolve as

$$
\begin{aligned}
|g, m, n\rangle \Rightarrow & \cos \left[\Omega F_{m-k, m}^{L} t\right]|g, m, n\rangle \\
& -i \exp \left(-i \phi_{2}\right) \sin \left[\Omega F_{m-k, m}^{L} t\right]|e, m-k, n\rangle, \text { for } m \geq k ; \\
|g, m, n\rangle \Rightarrow & |g, m, n\rangle, \text { for } m<k \\
|e, m, n\rangle \Rightarrow & \cos \left[\Omega F_{m+k, m}^{L} t\right]|e, m, n\rangle \\
& -i \exp \left(i \phi_{2}\right) \sin \left[\Omega F_{m+k, m}^{L} t\right]|g, m+k, n\rangle .
\end{aligned}
$$

Here the matrix element $F_{m-k, m}^{L}$ stands for

$$
F_{m-k, m}^{L}=i^{k}\left[\prod_{i=0}^{k-1} \eta_{c} \sqrt{(m-i)}\right]\left\langle m-k\left|\hat{O}_{k}^{L}\right| m-k\right\rangle,
$$

and $F_{m, m-k}^{L}=\left(F_{m-k, m}^{L}\right)^{*}$.

Case III. Quantized field in resonance with $k^{\text {th }}$ blue shifted vibrational sideband, $\delta_{0 c}=-k \nu, k=1,3, . . \infty$.

Neglecting quickly vibrating terms the Hamiltonian reduces to

$$
\hat{H}_{3}=\hbar g\left[\sigma_{+} \hat{b}\left(i^{k-1} \eta_{c}^{k}\right) \hat{a}^{\dagger k} \hat{O}_{k}^{c}+\sigma_{-} \hat{b}^{\dagger}\left((-i)^{k-1} \eta_{c}^{k}\right) \hat{O}_{k}^{c} \hat{a}^{k}\right] .
$$

From the initial states $|g, m, n\rangle$, and $|e, m, n\rangle$, the system evolves as

$$
\begin{aligned}
|g, m, n\rangle \Rightarrow & \cos \left[g \sqrt{n} F_{m+k, m}^{c} t\right]|g, m, n\rangle \\
& -i \sin \left[g \sqrt{n} F_{m+k, m}^{c} t\right]|e, m+k, n-1\rangle, \\
|e, m, n\rangle \Rightarrow & \cos \left[g \sqrt{n+1} F_{m-k, m}^{c} t\right]|e, m, n\rangle \\
& -i \sin \left[g \sqrt{n+1} F_{m-k, m}^{c} t\right]|g, m-k, n+1\rangle \text { for } m \geq k ; \\
|e, m, n\rangle \Rightarrow & |e, m, n\rangle, \text { for } m<k,
\end{aligned}
$$

with the matrix element, $F_{m-k, m}^{c}=\left\langle m-k\left|i^{k-1} \eta_{c}^{k} \hat{O}_{k}^{c} \hat{a}^{k}\right| m\right\rangle$ given by

$$
F_{m-k, m}^{c}=\left(i^{k-1}\right)\left[\prod_{i=0}^{k-1} \eta_{c} \sqrt{(m-i)}\right]\left\langle m-k\left|\hat{O}_{k}^{c}\right| m-k\right\rangle .
$$

For the special case $k=1$,

$$
F_{m-1, m}^{c}=\exp \left(-\frac{\eta_{c}^{2}}{2}\right) \eta_{c} \sqrt{m} \sum_{p=0}^{m-1} \frac{\left(i \eta_{c}\right)^{2 p}(m-1) !}{(p+1) ! p !(m-1-p) !} .
$$

We notice that for a given value of $\eta_{c}$, the characteristic frequencies, $\left[g \sqrt{(n+1)} F_{m-k, m}^{c}\right]$ and $\left[g \sqrt{n} F_{m+k, m}^{c} t\right]$ vary with the number of initial state vibrational and quantized field quanta. Choosing $m, n$ and $\eta_{c}$ to give large value for the matrix element $F_{m-k, m}^{c}$, can reduce the time required for implementing a quantum logic operation involving states $|e, m, n\rangle$ and $|g, m-k, n+1\rangle$. We may recall here that with the center of trap close to standing wave anti node, transitions involve state change by $k$ quanta, where $k=0,2,4, \ldots, \infty$.

Implementation of quantum logic gates

A Hadamard gate rotates single qubit states as below 


$$
|0\rangle \Longrightarrow \frac{|0\rangle+|1\rangle}{\sqrt{2}}, \quad|1\rangle \Longrightarrow \frac{|0\rangle-|1\rangle}{\sqrt{2}}
$$

A controlled-NOT gate on the other hand transforms two-qubit states as

$$
\begin{aligned}
|0,0\rangle & \Longrightarrow|0,0\rangle, \quad|0,1\rangle \Longrightarrow|0,1\rangle, \\
|1,0\rangle & \Longrightarrow|1,1\rangle, \quad|1,1\rangle \Longrightarrow|1,0\rangle,
\end{aligned}
$$

i.e., target qubit changes (does not change) state if the control qubit (vibrational state) has value one (zero). An application of a Hadamard gate, followed by a phase gate and another application of a Hadamard gate results in the implementation of a controlled-NOT gate. The phase gate involving a special phase shift of $\pi$, results in the following elementary operation,

$$
\begin{aligned}
|0,0\rangle & \Longrightarrow|0,0\rangle, \quad|0,1\rangle \Longrightarrow|0,1\rangle, \\
|1,0\rangle & \Longrightarrow|1,0\rangle, \quad|1,1\rangle \Longrightarrow-|1,1\rangle .
\end{aligned}
$$

With the cavity initially prepared in the vacuum state consider the initial states $|g, m, 0\rangle$ and $|e, m, 0\rangle$. The internal state of the ion is the target qubit whereas state of center of mass motion acts as control qubit. Choosing $\delta_{0 L}=0$, $\phi_{1}=\frac{\pi}{2}+2 \pi p,(p=1,2 .$.$) and applying a laser pulse for time t_{m, m}$, such that $\left[\Omega F_{m, m}^{L} t_{m, m}\right]=\frac{\pi}{4}$, we get from Eqs. (8 and 9)

$$
\begin{aligned}
& |g, m, 0\rangle \Rightarrow \frac{1}{\sqrt{2}}(|g, m, 0\rangle-|e, m, 0\rangle), \\
& |e, m, 0\rangle \Rightarrow \frac{1}{\sqrt{2}}(|e, m, 0\rangle+|g, m, 0\rangle) .
\end{aligned}
$$

This is the first step to implement a Hadamard gate. Next, a non-resonant pulse with $\delta_{0 L}=k \nu, k=1,2 \ldots$, interacting with the ion for time, $t_{m+k, m}=\pi /\left(\Omega F_{m+k, m}^{L}\right)$, may be used to switch the sign of state $|e, m, 0\rangle$ for $m<k$, as verified from Eqs. (11- 13) and shown by Wei et al [6]. Tuning the quantized cavity field to $k^{\text {th }}$ blue shifted vibrational sideband by choosing $\delta_{0 c}=-k \nu, k=1,3, . . \infty$, for an interaction time, $t_{m-k, m}=\pi /\left(g F_{m-k, m}^{c}\right)$, switches the sign of state $|e, m, 0\rangle$ with $m \geq k$ (Eqs. (16 and 17). The end result of this two step process is implementation of a Hadamard gate,

$$
\begin{aligned}
& |g, m, 0\rangle \Rightarrow \frac{1}{\sqrt{2}}(|g, m, 0\rangle+|e, m, 0\rangle), \\
& |e, m, 0\rangle \Rightarrow \frac{1}{\sqrt{2}}(|g, m, 0\rangle-|e, m, 0\rangle) .
\end{aligned}
$$

As the dependence of the matrix elements $F_{m, m}^{L}, F_{m+k, m}^{L}$ and $F_{m-k, m}^{c}$ on Lamb-Dicke parameters varies with $m$ and $k$, these choices determine the implementation time for Hadamard gate, which is $t_{H}=t_{m, m}+t_{m+k, m}$ for $m<k$, and $t_{H}=t_{m, m}+t_{m-k, m}$ for $m \geq k$.

To implement the phase gate, choose $\delta_{0 c}=-k \nu$, and the interaction time of the ion with the cavity field to be $t_{P}=\pi /\left(g F_{m-k, m}^{c}\right)$. From Eqs. (16-18), we find that

$$
\begin{aligned}
& |g, m, 0\rangle \Longrightarrow|g, m, 0\rangle ; \quad \text { for all } m, \\
& |e, m, 0\rangle \Longrightarrow|e, m, 0\rangle ; \quad \text { for } m<k, \\
& |e, m, 0\rangle \Longrightarrow-|e, m, 0\rangle ; \quad \text { for } m \geq k,
\end{aligned}
$$

or the phase gate of Eq. (23) is implemented. The quantized cavity field states serve as the auxiliary qubit in the implementation of phase gate required to implement a controlled-NOT quantum logic gate. An important observation is that the the cavity is initially in the vacuum state and is left in the vacuum state in the end [14]. As such successive quantum logic gates can be implemented even in the presence of cavity losses.

The speed of operation, in ionic state manipulation, is extremely important for successful experimental realization. The operation time should not be so long as to allow the decoherence due to interaction with environment to dominate the scene. To analyze the switching times, we consider the control-bit vibrational phonons to take values $m$ and $m-1$ 
( $m=1,4,9$, and 16). Table I and II list the expected ion-laser/quantized field interaction times $t_{m, m}$ and $t_{m-1, m}$ for $\Omega=g=2 \pi \times 140 \mathrm{kHz}$ and the choices $\eta_{L}=\eta_{c}=0.02$ (LD regime), and 0.2 (beyond LD regime). Experimentally cold ion traps with parameter values given above have been realized successfully [2] and ions in vibrational state with $m$ up to 16 obtained [3]. In experiments performed with trapped Calcium ions and optical cavities [12], ion-cavity coupling $g \approx 6 \mathrm{MHz}$.

In the Lamb-Dicke regime $\left(\eta_{L}=\eta_{c}=0.02\right)$, the characteristic time, $t_{m, m}^{1}$, needed during the realization of a Hadamard gate is independent of $m$ since $F_{m, m} \approx 1$. Working beyond the Lamb-Dicke limit $\left(\eta_{L}=\eta_{c}=0.2\right.$ here), $F_{m, m}<1$, causes an increase in $t_{m, m}^{2}$ with increasing $m$. The last column in table I shows that for $m=16, t_{16,16}^{2}$ is twice as large as $t_{1,1}^{1}$. We may consider $2 \mu s$ to be the characteristic time for the step involving interaction of ion with resonant laser pulse.

The characteristic time, $t_{m-1, m}=\pi /\left(g F_{m-1, m}\right)$, for implementing ionic state transition using off resonance laser pulse or quantized cavity field, is seen to decrease with increase in the number of initial state quanta $m$, for $\eta_{L}=$ $\eta_{c}=0.02$, as well as 0.2. In addition for the case $\eta_{L}=\eta_{c}=0.2$, that is going out from LD regime, $t_{m-1, m}$ shows a sharp decline for all $m$ values considered. In general for a given $m$ value $t_{m-1, m}$ is larger in comparison with $t_{m, m}$. Usually the control qubit is encoded by vibrational quanta 0 and 1 . We notice however that working beyond the LD regime, large $m$ values result in shorter times for ionic state manipulation. For example the choice $m=16$, results in $t_{0,1}^{1} / t_{15,16}^{2}=28.5$. It is exciting to think that by choosing hotter initial ionic states, the state manipulation time can come down from $\approx 178 \mu s$ to $\approx 6 \mu s$. We conclude that faster ionic state manipulation for implementing quantum logic gates is achieved in ion trap - optical cavity system, by working beyond the Lamb-Dicke regime rather than in Lamb-Dicke regime. Recently a coherence time of $\approx 1 \mathrm{~ms}$ for qubits based on single $\mathrm{Ca}^{+}$ion has been reported [16]. We must recall however that the optical cavity decay time is, $\tau_{c} \approx 2 \mu s[13,15]$. In comparison, the ionic state manipulation times for operations involving change of cavity state, are still quite large and much needs to be done in the experimental field.

Acknowledgement 1 S.S.S would like to thank Unicamp for hospitality. This work is partially supported by CNPq, Brazil.

[1] A. Barenco, C. H. Bennet, R. Cleve, D. P. DiVincenzo, N. Margolus, P. Shor, T. Sleater, J. A. Smolin, and H. Weinfurter, Phys. Rev. A 52, 3457 (1995).

[2] C. Monroe, D. M. Meekhof, B. E. King, S. R. Jefferts, W. M. Itano, and D. J. Wineland, Phys. Rev. Lett., 75, 4011 (1995).

[3] D.J. Wineland, C. Monroe, W.M Itano, D. Leibfried, B.E. King, and D.M. Meekhof, NIST J. Res. 103, 259 (1998).

[4] J.I. Cirac and P. Zoller, Phys. Rev. Lett., 74, 4091 (1995).

[5] L. F. Wei, S. Y. LIU and X. L. Lei, Phys. Rev. A 65, 062316 (2002).

[6] L. F. Wei, S. Y. LIU and X. L. Lei, Opt. Commun., 208, 131(2002).

[7] A. Rauschenbeutel, G. Nogues, S. Osnaghi, P. Bertet, M. Brune, J.M. Raimond, and S. Haroche, Phys. Rev. Lett. 83, 5166 (1999).

[8] A.S. Parkins and H.J. Kimble, J. Opt. B: Quantum Semiclass Opt. 1, 496 (1999)

[9] F. L. Semião, A. Vidiella-Barranco, J. A. Roversi, Phys. Rev. A 64, 024304 (2001).

[10] J. Pachos and H. Walther, Phys. Rev. Lett. 89, 187903 (2002).

[11] E.Jane, M.B. Plenio and D. Jonathan, Phys. Rev. A 65 (2002) 050302(R).

[12] G.R. Guthöhrlein, M. Keller, K. Hayasaka, W. Lange, and H. Walther, Nature, 41449 (2001).

[13] A. B. Mundt, A. Kreuter, C. Becher, D. Liebfried, J. Eschner, F. Schmidt-Kaler, and R. Blatt, Phys. Rev. Lett. 89, 103001 (2002).

[14] F. L. Semião, A. Vidiella-Barranco, J. A. Roversi, Phys. Lett. A 299, 423 (2002).

[15] B.T.H. Varcoe, S. Brattke, M. Weldinger, and H. Walther, Nature 403743 (2000).

[16] F. Schmidt-Kaler, S. Gulde, M. Riebe, T. Deuschle, A. Kreuter, G. Lancaster, C. Becher, J. Eschner, H. Hffner, and R. Blatt, J. Phys. B: At. Mol. Opt. Phys. 36, 623636 (2003). 
TABLE I. Characteristic times, $\left(t_{m, m}=\pi /\left(4 \Omega F_{m, m}\right)\right)$, and matrix elements $F_{m, m}$ for implementing ionic state transitions using resonant laser pulse/ quantized cavity field, for Rabi frequency, $\Omega=g=2 \pi \times 140 \mathrm{kHz}$.

\begin{tabular}{cccccc}
\hline \hline & LD regime, $\eta_{L}=\eta_{c}=0.02$ & & Beyond LD regime, $\eta_{L}=\eta_{c}=0.2$ \\
$m$ & $F_{m, m}$ & $t_{m, m}^{1}(\mu s)$ & $F_{m, m}$ & $t_{m, m}^{2}(\mu s)$ & 0.9 \\
1 & 0.999 & 0.90 & 0.941 & 0.95 & 0.8 \\
4 & 0.998 & 0.90 & 0.828 & 1.07 & 0.3 \\
9 & 0.996 & 0.90 & 0.654 & 2.02 & 0.5 \\
16 & 0.993 & 0.90 & 0.441 & $R_{m, m}^{1}$ & $t_{1,1}^{2}$ \\
\hline \hline
\end{tabular}

TABLE II. Characteristic times, $\left(t_{m-1, m}=\pi /\left(g F_{m-1, m}\right)\right)$, and matrix elements $F_{m-1, m}$ for implementing ionic state transitions using off resonance laser pulse/ quantized cavity field for Rabi frequency, $\Omega=g=2 \pi \times 140 k H z$.

\begin{tabular}{cccccc}
\hline \hline & LD regime, $\eta_{L}=\eta_{c}=0.02$ & & Beyond LD regime, $\eta_{L}=\eta_{c}=0.2$ \\
$m$ & $F_{m-1, m}$ & $t_{m-1, m}^{1}(\mu s)$ & $F_{m-1, m}$ & $t_{m-1, m}^{2}(\mu s)$ & $R_{m-1, m}=\frac{t_{0,1}^{1}}{t_{m-1, m}^{2}}$ \\
1 & 0.02 & 178.57 & 0.196 & 18.22 & 9.8 \\
4 & 0.04 & 89.28 & 0.369 & 9.68 & 18.4 \\
9 & 0.06 & 59.52 & 0.498 & 7.17 & 24.9 \\
16 & 0.08 & 44.64 & 0.570 & 6.26 & 28.5 \\
\hline \hline
\end{tabular}

